\title{
Os Dividendos Como Estratégia de Investimentos em Ações*
}

\author{
Artur Franco Bueno \\ Mestre em Controladoria e Contabilidade pela FEA/USP \\ Gerente Financeiro da RTC-Brasil
}

RESUMO

$\mathrm{Na}$ Teoria de Finanças, o papel dos dividendos nas cotações é um tema controverso, uma vez que há diferentes teorias que abordam sua relevância para o valor das ações e, em conseqüência, para a riqueza dos acionistas.

Além disso, as descobertas empíricas que originaram a Hipótese de Mercado Eficiente de Capitais (HME) não apenas contradizem a Análise Técnica, mas também impõem um desafio à declarada habilidade superior da Análise Fundamentalista em gerar retornos superiores, com base em fatores ou variáveis contábeis, financeiros e econômicos, tais como o dividend yield (retorno em dividendos).

A fim de se verificar a relação entre dividend yieldse as taxas de retornos das ações, bem como a viabilidade de uma estratégia baseada em dividend yields históricos de "bater" o mercado, foram construídas, mensalmente, durante o período que vai do Plano Real em julho de 1994 a dezembro de 1999, três diferentes carteiras (alto, baixo e zero). Seus riscos, retornos e indicadores de desempenho ajustados ao risco foram calculados e comparados entre si e com seu paradigma (benchmark), o Índice da Bolsa de Valores de São Paulo - Ibovespa.

A evidência empírica é incapaz de sugerir que as ações de altos dividend yield tendem a possuir maiores ou menores taxas de retorno do que as ações de baixo ou zero yield.

Ademais, as evidências sugerem que não é possível demonstrar, usando o método empírico aplicado, uma clara associação entre dividend yield e taxas de retorno das ações.

Palavras-chave: mercado de capitais, dividendos, ações, investimentos.
ABSTRACT

In Finance Theory, the role of dividends in stock prices is a controversial issue, considering the different theories that exist on their relevance for stock value and, consequently, for shareholders' wealth.

Furthermore, empirical findings that have originated the Efficient Market Hypothesis (EMH) not only contradict Technical Analysis, but also pose a challenge to the Fundamental Analysis' supposedly superior ability to generate higher returns based on accounting, financial and economic factors or variables such as the dividend yield.

In order to assess the relationship between dividend yields and stocks returns as well as the viability of a strategy based on historic dividend yield to "beat" the market, with reference to the period from the beginning of the Real Plan in July 1994 until December 1999, three different yield portfolios (high, low and zero) were formed on a monthly basis. Their risks, returns and risk-adjusted portfolio performance measurements were calculated, and then compared both among themselves and to the benchmark (São Paulo Stock Exchange Index - Ibovespa).

Empirical evidence is unable to suggest either that stocks with a high dividend yield tend to have higher or lower returns than those with a low or zero yield.

Moreover, the evidences suggest that, using the applied empirical method, no clear association between dividend yield and stock returns can be demonstrated.

Key words: capital markets, dividends, stocks, investments.

* O presente artigo corresponde a um resumo da dissertação intitulada “Análise Empírica do Dividend Yield das Ações Brasileiras", defendida na FEA/USP 


\section{INTRODUÇÃO}

Tão antigos quanto as próprias bolsas de valores são os estudos ou modelos (stock valuation models) que almejam descobrir estratégias de investimento em ações de modo a formarem-se carteiras capazes de oferecerem retornos superiores aos índices de mercado.

O que tais modelos tentam revelar, em essência, é a ocorrência de má precificação dos preços das ações pelo mercado, o que permite ao investidor "ganhar" do mercado através da incorporação ou eliminação ex-ante das ações que porventura fossem identificadas como subavaliadas ou sobrevalorizadas, respectivamente.

Os primeiros a defenderem o fluxo de dividendos como critério de seleção de ações foram Graham e Dodd, dois expoentes da Tradicional Teoria de Portfólios, no início da década de 1930.

Uma das estratégias desenvolvidas a partir de então foi a seleção de ações por meio das taxas de retorno em dividendos de uma ação, doravante denominada dividend yield (mas também conhecida como cash yield ou cash dividend yield).

A utilização do dividend yield nos EUA é de tal magnitude que este foi um dos indicadores utilizados para se corroborar a tese de que o mercado acionário norte-americano vivia um período de "exuberância irracional'", isto é, as ações estariam tão sobrevalorizadas (caras) que o risco de abrupta correção ou crash era iminente. Parte desta preocupação foi sugerida pelo fato de que o índice dividendos/preço ou o dividend yield havia decaído a seus níveis mais baixos e, portanto, isso implicaria que os preços das ações estariam muito elevados (KRAMER, 1997:24) ${ }^{2}$.

Se, à primeira vista, parece ser inquestionável a relevância dos dividendos, de acordo com o que foi descrito, além do fato de influenciar as decisões estratégicas fundamentais no cotidiano das empresas, trata-se, ao contrário, de tema ainda bastante contro- verso para as Finanças.

Os dividendos não são a única fonte de recursos do fluxo de caixa dos investidores. Há também o ganho de capital decorrente da venda de suas ações. A partir da diferença de tributação a favor dos ganhos de capital, foi criada a Teoria da Preferência Tributária que defende uma política de baixos dividendos a fim de que sejam maximizados os valores das ações.

Não obstante, tendo como fulcro sua teoria sobre estrutura de capital, em outro trabalho revolucionário, conhecido como Teoria da Irrelevância dos Dividendos, MILLER \& MODIGLIANI - M\&M (1961) demonstraram também que pode haver indiferença na distribuição de grandes ou pequenos montantes de dividendos, o que torna este tópico, na opinião de ROSS et al. (1995: 389), "fascinante, pois é equivalente a um quebra-cabeças". Obviamente, esta terceira corrente lançou mais dúvidas sobre o tema, mas, por outro lado, atribuiu nova motivação às pesquisas empíricas.

Não fosse o bastante, em idos da década de 1960, importantes descobertas empíricas acerca da aleatoriedade dos preços das ações, como os artigos de ROBERTS (1959) e de OSBORNE (1959), bem como a redescoberta da Teoria da Especulação escrita em 1900 por Louis Bachelier (BERNSTEIN, 1992: 23), obrigaram a uma revisão da Teoria Econômica a respeito das flutuações nos preços das ações.

Dessa revisão teórica surgiu a Hipótese de Mercados Eficientes de Capitais - $\mathrm{HME}^{3}$, isto é, a crença no dogma de que os preços de mercado das ações refletem as informações disponíveis. Contrariamente à idéia de que existem oportunidades para se auferirem ganhos extraordinários ou anormais (maiores que o retorno do próprio mercado) e, consequentemente, que seja possível desenvolver modelos ou estratégias (entre elas a do dividend yield) a fim de identificar imperfeições nas cotações de mercado das ações, essa corrente defende justamente o oposto, ou seja, a quase impossibilidade de, com base nas informações públicas disponíveis, contabilizarem-se retornos anormais.

\footnotetext{
${ }^{1}$ Em um de seus discursos públicos em 1997, Alan Greenspan, então presidente do Federal Reserve, o Banco Central dos EUA, cunhou o termo irrational exuberance para justificar a euforia da Bolsa de Valores de Nova Iorque.

$2 \mathrm{O}$ autor sugere que o mercado de ações estaria vivenciando um novo patamar e, por conseguinte, o baixo dividend yield poderia ser decorrente de outros fatores que não a sobrevalorização em si, mas, principalmente, o crescente ingresso de recursos às bolsas via fundos mútuos de investimentos.

3 A fusão da HME com a extensão do Modelo de Markowitz, o Modelo de Precificação de Ativos - CAPM resultaram na chamada Moderna Teoria de Portfólios (ou Carteiras) - MTP.
} 


\section{ESTUDOS EMPÍRICOS}

Como, de um lado, as opiniões a respeito da influência dos dividendos sobre as cotações ficaram divididas desde o clássico artigo de $M \& M$, e, de outro lado, a comprovação ou refutação da HME representava um desafio adicional, vários estudos foram realizados.

Os resultados parecem conduzir os pesquisadores a um beco sem saída. GITMAN (1997: 517) observou que "embora muitos estudos já tenham sido realizados com vistas a validar ou refutar a teoria da irrelevância dos dividendos, nenhum deles alcançou uma evidência irrefutável".

Enquanto BLACK \& SCHOLES (1974), MILLER \& SCHOLES (1982) e MEGLIORINI (1997) não notaram nenhuma relação significativa entre retornos e dividendos, BRIGHAM \& GORDON (1968) e LONG JR. (1978) não confirmaram a irrelevância dos dividendos, e HESS (1982) acrescentou que a relação é inconsistente com o conteúdo informacional e o efeito clientela ${ }^{4}$. Além disso, os estudos de LITZENBERGER \& RAMASWAMY (1979, 1980, 1982), BLUME (1980) e ELTON et al. (1983) sugerem haver uma relação entre retornos esperados $e$ dividendos.

MORGAN (1982) chega a sugerir uma relação nãolinear entre dividendos e retornos, ao passo que KEIM (1985) aponta para uma concentração dessa relação no mês de janeiro.

Por outro lado, FRIEND \& PUCKETT (1964), POTERBA (1986) e SPERANZINI (1994) não compartilham a preferência por dividendos.

Visto que a não-linearidade parece ser inconsistente com o efeito imposto ${ }^{5}$, foi levantada a hipótese de que a relação entre retornos e dividend yield pode ser dirigida por diferenças no risco (Summers apud CHRISTIE, 1990: 96-97). Uma vez que o valor dos dividendos é relativamente estável, as mudanças no risco serão mais sentidas nos preços do que nos dividendos, o que implica uma "relação induzida pelo risco positivo entre os retornos esperados ex ante e os dividend yields não relacionados ao efeito imposto. $\mathrm{A}$ não-linearidade poderia, então, levantar a questão se companhias de dividend yield igual a zero possuem risco maior àquelas de baixo dividend yield ". Foi o que Keim (op. cit.) apresentou.

Outra explicação oferecida por ELTON et al. (1983) sugere que, como as ações de zero dividend yield são, em geral, negociadas a US\$ 5/ação (não podendo, portanto, servirem para chamadas de margem), elas oferecem maiores retornos antes dos impostos se comparadas às ações de baixo dividend yield, a fim de induzirem os investidores a mantêlas em carteira.

O fato de a questão permanecer em aberto até hoje mereceu o seguinte comentário de ROSS et al. (1995: 387):

É surpreendente que os resultados de tanta pesquisa de qualidade uniformemente elevada possam ser tão conflitantes. Pode-se apenas esperar que as ambigüidades sejam elucidadas no futuro. Infelizmente, a formulação de estratégias ótimas de investimento para os indivíduos ainda não é fácil dada a confusão existente.

$\mathrm{Na}$ verdade, os pesquisadores não podem ainda dizer aos profissionais como a política de dividendos afeta o custo de capital nem o valor das ações, pois as pesquisas feitas a fim de responderem a questão sobre a importância dos dividendos não lograram sucesso de forma cabal. KEOWN et al. (1996: 592-593) ponderam que "para testar a relação entre o pagamento de dividendos e cotação das ações poderíamos comparar o retorno em dividendos de uma companhia e seu retorno. A questão é: as ações que pagam os maiores dividendos produzem os maiores ou os menores retornos?".

Segundo os autores, ambos os resultados foram encontrados, com uma ligeira vantagem para as ações de baixos dividendos, mas nada conclusivo, devido ao alto desvio padrão das estimativas estudadas, o que sugere ser o resultado de erro aleatório da amostra e não realmente diferenças. "Nós simplesmente temos sido incapazes de "desembaraçar" o efeito da política de dividendos das outras influências" (ibid.), concluem.

A falta de capacidade para "desembaraçar o nó" desse problema tem várias razões. KEOWN et al.

\footnotetext{
${ }^{4}$ O leitor não familiarizado com tais teorias pode encontrar alguns referenciais em BUENO (2000). 
(1996:593) levantam dois pontos: primeiro, para se ter acurácia, deve-se ter a quantia esperada pelos investidores acerca dos dividendos. Visto que estas expectativas não podem ser observadas, são utilizados dados históricos que podem ou não estar relacionados com as expectativas. Em segundo lugar, a maioria dos estudos assume uma relação linear entre dividendos e cotações, enquanto a verdadeira relação pode ser não-linear e possivelmente descontínua.

WESTON \& BRIGHAM (2000: 711) acrescentam que o maior problema das pesquisas com base numa perspectiva do CAPM "é que os pesquisadores em geral utilizam taxas históricas de retorno como substitutos para os retornos exigidos, e com esses dados inadequados, era quase inevitável que os testes tivessem resultados controversos".

Já que as pesquisas conduzidas por profissionais carecem de rigor científico, e os testes estatísticos ainda não podem fornecer evidências conclusivas, KEOWN et al. (1996: 593) fizeram uma última tentativa: com base no estudo de Baker, Farrelly e Edelman, de 1983, procuraram respostas nos questionários respondidos pelos administradores norte-americanos, pois, ainda que a conclusão não seja a palavra final, podem prover algumas revelações (insights) importantes. Os resultados foram os seguintes:

$\checkmark$ existe uma evidência - não soberana - a favor da relevância da política de dividendos (em sua maioria estão divididos entre os que julgam que os dividen- dos são importantes e os que não têm opinião formada a respeito);

$\checkmark$ a maioria também acredita que os dividendos afetam as cotações;

$\checkmark$ sobre o conteúdo informacional, houve divisão entre os que concordam e os que não possuem opinião sobre se os dividendos trazem informações sobre o futuro das empresas;

$\checkmark$ quase $2 / 3$ acreditam que os investidores têm preferência por dividendos ou ganhos de capital, mas somente $56 \%$ acreditam que os investidores percebam diferença no grau de risco entre as duas formas de retorno;

$\checkmark$ a maioria também não considera que os acionistas são atraídos porque as políticas de dividendos são apropriadas aos seus planejamentos fiscais, nem crêem no conceito de efeito clientela.

\section{RESULTADOS DA PESQUISA}

O período compreendido pela pesquisa (julho/1994 a dezembro/1999), além de conservar um padrão de conjuntura econômica, conteve nítidos ciclos de alta e de baixa da Bovespa, algo praticamente inviável de ser observado em prazos inferiores a dois anos.

Dentre os principais eventos político-econômicos que ratificaram ou reverteram tendências de alta ou de baixa do lbovespa no período destacam-se os seguintes (tabela 3.1):

Tabela 3.1 - Eventos Político-econômicos 1994-1999

\begin{tabular}{|c|c|}
\hline Julho / 1994 & Nova moeda - Plano Real, como seqüência da URV lançada em março \\
\hline Outubro / 1994 & Eleições presidenciais no Brasil \\
\hline Dezembro / 1994 & $\begin{array}{l}\text { Crise da balança de pagamentos do México e decretação de Regime de Administração Especial } \\
\text { Temporária - RAET no Banespa }\end{array}$ \\
\hline Agosto / 1995 & Intervenção do BACEN no Banco Econômico \\
\hline Novembro / 1995 & Decretado o RAET no Banco Nacional \\
\hline Julho / 1997 & Crise cambial da Tailândia que se alastrou aos ex-tigres asiáticos nos meses seguintes \\
\hline Maio / 1998 & Liberada a participação de capital estrangeiro na privatização da Telebrás (ocorrida em julho) \\
\hline Outubro / 1998 & Eleições presidenciais no Brasil \\
\hline Agosto / 1998 & Crise da moratória russa \\
\hline Janeiro / 1999 & Desvalorização do real \\
\hline Fevereiro / 1999 & Toma posse o novo presidente do BC Armínio Fraga \\
\hline Março / 1999 & $\begin{array}{l}\text { Revisão do acordo celebrado em dezembro / } 1998 \text { com o FMI, que resultou na liberação da } 2^{a} \text { parcela } \\
\text { do empréstimo }\end{array}$ \\
\hline
\end{tabular}

Fontes: Bradespar e Laboratório de Finanças FIA/FEA/USP. 
Inicialmente, os resultados (tabela 3.2 e gráfico 3.1) revelam que a carteira de zero yield obteve o maior retorno acumulado $(233,6 \%)$, o maior retorno médio mensal $(2,7 \%)$ e, também, o maior risco ou desvio padrão $(13,4 \%)$.

Gráfico 3.1 - Evolução das Carteiras (jul/1994=100)

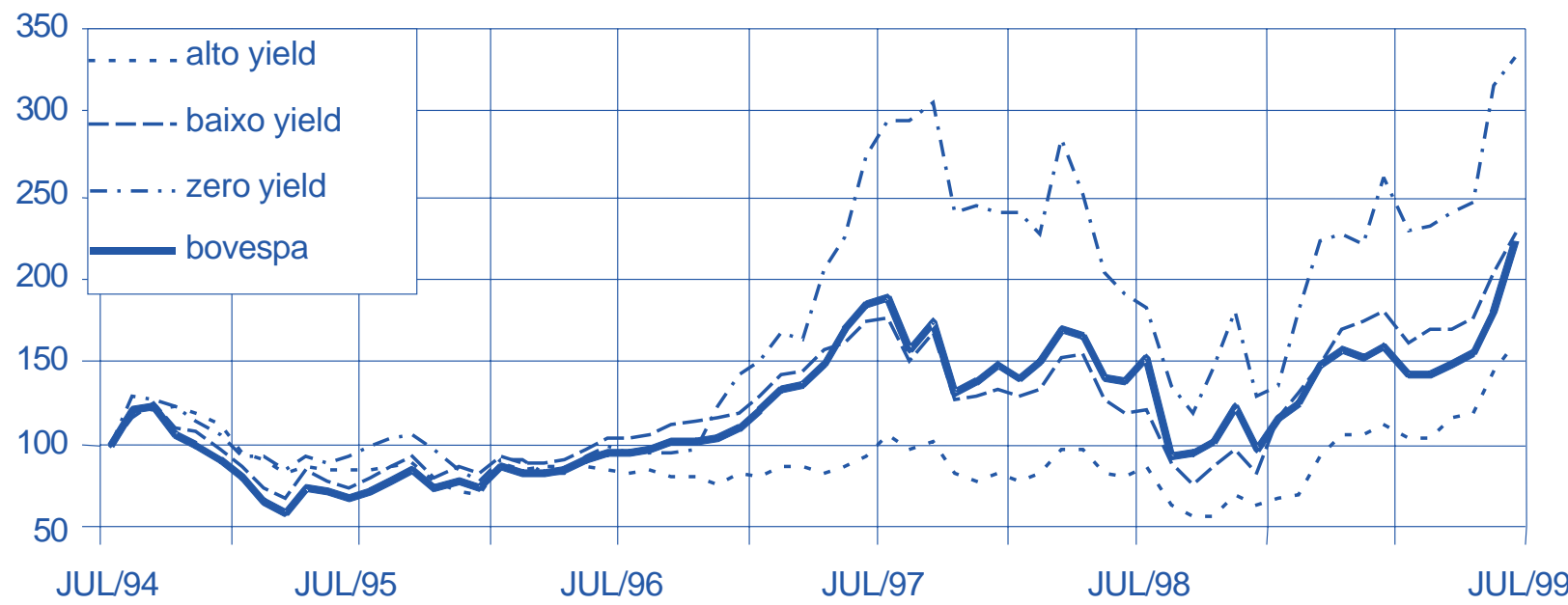

A carteira de menor retorno acumulado e, consequentemente, média mensal, foi a de alto yield, $61,7 \%$ no período e $1,3 \%$ a.m. Por outro lado, foi a carteira de menor risco (desvio padrão de 10,7\%) no período.

A carteira de baixo yield e o lbovespa situaram-se em posições intermediárias (retorno acumulado de $128,5 \%$ e $123,5 \%$, respectivamente), sendo que a primeira contabilizou o maior retorno em um único mês (42,9\% em janeiro/1999) e a outra a maior queda ($39,2 \%$ em agosto/1998).

Tabela 3.2 - Estatística Descritiva

\begin{tabular}{|l|r|r|r|r|}
\hline \multicolumn{1}{|c|}{$\%$} & Alto Yield & Baixo Yield & Zero Yield & Ibovespa \\
\hline Acumulado & 61,7 & 128,5 & 233,6 & 123,5 \\
\hline Média Mensal & 1,3 & 1,9 & 2,7 & 2,0 \\
\hline Variância Mensal & 1,1 & 1,3 & 1,8 & 1,4 \\
\hline Desvio padrão Mensal & 10,7 & 11,5 & 13,4 & 12,0 \\
\hline Coeficiente de Variação & 832,6 & 597,6 & 491,6 & 600,3 \\
\hline Máximo & 34,4 & 42,9 & 32,9 & 25,6 \\
\hline Mínimo & $-26,2$ & $-26,9$ & $-28,1$ & $-39,2$ \\
\hline
\end{tabular}

Entrementes, ao se cotejarem os coeficientes de variação, observa-se que justamente a carteira de alto yield possui a maior dispersão relativa ( 8,3 ou $832,6 \%$ ), logo, o maior risco em relação ao retorno. Por outro lado, a carteira de zero yield foi a de melhor desempenho no período, de acordo com o coeficiente de variação (4,9 ou $491,6 \%$ ), ficando a carteira de baixo yield e o lbovespa nas posições intermediárias (597,6\% e 600,3\%).
Os cálculos do teste t de Student para duas amostras, presumindo-se variância diferentes (tabela 3.3), por seu turno, não foram capazes de rejeitar $\mathrm{H}_{0}$, ou seja, as diferenças de médias não foram estatisticamente significativas. Contudo, vale advertir que a grande variabilidade para as três carteiras pode ter comprometido a detecção de diferenças significativas entre elas. 
Tabela 3.3 -Teste t para 2 Amostras (Variâncias Diferentes) jul/1994-dez/1999

\begin{tabular}{|l|c|c|c|c|}
\hline P-value bicaudal & Alto Yield & Baixo Yield & Zero Yield & Ibovespa \\
\hline Alto Yield & & & & \\
\hline Baixo Yield & 0,75 & & & \\
\hline Zero Yield & 0,50 & 0,71 & & \\
\hline Ibovespa & 0,72 & 0,97 & 0,74 & \\
\hline
\end{tabular}

Como era de se esperar, os $p$-values entre as carteiras de desempenho extremos (alto e zero) registraram o menor nível (embora ainda elevado) de significância $(0,50)$, enquanto entre a carteira de baixo yield e o lbovespa, de desempenho muito próximos, foi verificado o maior $p$-value $(0,97)$.

Após a aplicação de testes t para duas amostras, presumindo-se variâncias diferentes para as quatro carteiras em pares, que não foi capaz de rejeitar a hipótese nula, partiu-se para a análise dos 11 semestres, conforme descrito no capítulo anterior.

Lembrando que, diferentemente do teste aplicado ao período total (65 meses), quando se compararam as taxas médias de retornos mensais das próprias carteiras, nesta fase, em que os testes foram aplicados a subperíodos de 6 meses, foram comparadas as taxas semestrais de retornos das ações que compunham, com pesos iguais, as diferentes carteiras.

Para tanto, foram excluídas as ações que não se mantiveram na carteira pelos seis meses do subperíodo correspondente. Por exemplo, no segundo semestre de 1994, das ações que faziam parte da carteira de alto yield em julho, Brahma PN e Banco Itaú PN foram descartadas para fins de aplicação dos testes, uma vez que foram substituídas na carteira ao longo dos seis meses. Desta forma, somente oito ações (para a carteira de alto yield) foram testadas naquele subperíodo.

Tabela 3.4 - Quantidade de Ações Testadas por Carteira

\begin{tabular}{|c|c|c|c|c|c|c|c|}
\hline Ações Avaliadas & Ações em & \multicolumn{2}{|c|}{ Alto Yield } & \multicolumn{2}{|c|}{ Baixo Yield } & \multicolumn{2}{|c|}{ Zero Yield } \\
\hline 2ㅇ Semestre 1994 & & 8 & 10 & 7 & 10 & 7 & 7 \\
\hline 1ㅇ Semestre 1995 & & 5 & 10 & 5 & 10 & 4 & 5 \\
\hline 2 Semestre 1995 & & 8 & 10 & 7 & 10 & 6 & 6 \\
\hline 1ㅇ Semestre 1996 & & 6 & 10 & 5 & 10 & 4 & 7 \\
\hline 2 Semestre 1996 & & 8 & 10 & 6 & 10 & 7 & 7 \\
\hline 1ㅇ Semestre 1997 & & 4 & 10 & 4 & 10 & 6 & 7 \\
\hline 2 Semestre 1997 & & 6 & 10 & 7 & 10 & 4 & 4 \\
\hline 10 Semestre 1998 & & $\theta$ & 10 & 5 & 10 & $z$ & 3 \\
\hline 2 Semestre 1998 & & 7 & 10 & 8 & 10 & 3 & 3 \\
\hline 10 Semestre 1999 & & $\theta$ & 10 & Z & 10 & 3 & 3 \\
\hline 2 Semestre 1999 & & 5 & 10 & 5 & 10 & 4 & 6 \\
\hline
\end{tabular}

Obs.: as linhas tachadas significam que não foram realizados os testes nesses períodos por falta de elementos.

Para os primeiros semestres de 1998 e 1999, no entanto, os testes nem chegaram a ser aplicados, pois para a carteira zero e para a carteira baixo yield não foram obtidos elementos (ações) suficientes (tabela 3.4), denotando que, naqueles semestres, houve grande giro de posições naquelas carteiras.

A prova de condição de normalidade, de uma ma- neira geral, exibe níveis descritivos $p$ superiores a 0,10 (tabela 3.5), ou seja, o teste de aderência não rejeita a hipótese nula de normalidade dos dados (retornos) em um nível de significância de 10\%. Isto significa que as provas estatísticas baseadas na suposição de normalidade são aplicáveis.

As exceções foram a carteira de alto yield no $2^{\circ}$ 
semestre/1999 (0,0487); as carteiras de baixo yield no $2^{\circ}$ semestre/1994 $(0,018)$ e no $2^{\circ}$ semestre/1995 $(0,058)$; e a carteira de zero yield no $1^{\circ}$ semestre/1997 $(<0,01)$, no $2^{\circ}$ semestre/1997 $(0,0459)$ e no $2^{\circ}$ semestre/1999 (0,0404); que, com exceção do 1o semestre/
1997, ficaram bem próximas de um nível de significância de 5\%. Porém, como em nenhum momento a condição foi rejeitada para as três carteiras simultaneamente, os respectivos testes paramétricos foram aplicados.

Tabela 3.5 - Condição de Normalidade

\begin{tabular}{|c|c|c|c|c|c|c|}
\hline \multirow[t]{2}{*}{ Período / Carteira } & \multicolumn{3}{|c|}{ Nível Descritivo $p$} & \multicolumn{3}{|c|}{ Valor Tabulado } \\
\hline & alto & baixo & zero & alto & baixo & zero \\
\hline 2 Semestre 1994 & $>0,10$ & $0,018^{*}$ & $>0,10$ & 0,115 & 0,024 & 0,656 \\
\hline 10 Semestre 1995 & $>0,10$ & $>0,10$ & $>0,10$ & 0,533 & 0,781 & 0,526 \\
\hline 2o Semestre 1995 & $>0,10$ & $0,058^{*}$ & $>0,10$ & 0,596 & 0,039 & 0,468 \\
\hline 10 Semestre 1996 & $>0,10$ & $>0,10$ & $>0,10$ & 0,738 & 0,339 & 0,825 \\
\hline 2ㅇ Semestre 1996 & $>0,10$ & $>0,10$ & $>0,10$ & 0,596 & 0,123 & 0,214 \\
\hline 1ㅇ Semestre 1997 & $>0,10$ & $>0,10$ & $<0,01^{*}$ & 0,528 & 0,512 & 0,000 \\
\hline 2 Semestre 1997 & $>0,10$ & $>0,10$ & $0,0459^{*}$ & 0,315 & 0,801 & 0,055 \\
\hline 2o Semestre 1998 & $>0,10$ & $>0,10$ & $>0,10$ & 0,632 & 0,308 & 0,581 \\
\hline 2 Semestre 1999 & $0,0487^{*}$ & $>0,10$ & $0,0404^{*}$ & 0,059 & 0,197 & 0,052 \\
\hline
\end{tabular}

Obs.: $\left.{ }^{*}\right)$ como $p$-value $<0,10$, as hipóteses nulas foram rejeitadas.

Para a condição de homocedasticidade (tabela 3.6), novamente os níveis descritivos $p$ foram, em sua maioria, superiores a 0,10 e quase totalmente superiores a 0,05, à exceção do teste de Barlett para 1997.

Tabela 3.6 - Condição de Homocedasticidade

\begin{tabular}{|c|c|c|}
\hline P-value & Barlett & Levene \\
\hline 2 Semestre 1994 & 0,859 & 0,920 \\
\hline 10 Semestre 1995 & $0,064^{*}$ & 0,141 \\
\hline 2 Semestre 1995 & 0,426 & 0,473 \\
\hline 10 Semestre 1996 & $0,056^{*}$ & $0,064^{*}$ \\
\hline 2 Semestre 1996 & 0,285 & 0,448 \\
\hline 10 Semestre 1997 & $0,001^{*}$ & 0,622 \\
\hline 20 Semestre 1997 & $0,000^{*}$ & 0,180 \\
\hline 2o Semestre 1998 & $0,053^{*}$ & $0,079^{*}$ \\
\hline 2 Semestre 1999 & 0,428 & 0,778 \\
\hline
\end{tabular}

Obs.: (*) como $p$-value $<0,10$, as hipóteses nulas foram rejeitadas.

De uma maneira geral, os testes de Kruskal-Wallis - KW não rejeitaram a igualdade de retornos semestrais acumulados entre as três carteiras. $\mathrm{O}$ mesmo pode se afirmar da ANOVA $^{6}$, cujos resultados foram similares, ou seja, não se detectaram diferenças nos retornos entre as carteiras (tabela 3.7).

\footnotetext{
${ }^{6}$ Procedeu-se ao cálculo da Análise da Variância - ANOVA, pois foram verificadas as suposições de normalidade e homocedasticidade (teste de Levene e de Bartlett, quando aplicáveis).
} 
Tabela 3.7 - Resultados do Teste KW e ANOVA para as Médias das Carteiras

\begin{tabular}{|c|c|c|}
\hline P-value & Teste KW & ANOVA \\
\hline 2ㅇ Semestre 1994 & 0,503 & 0,653 \\
\hline 1ㅇ Semestre 1995 & 0,374 & 0,336 \\
\hline 2 Semestre 1995 & $0,020^{*}$ & $0,018^{*}$ \\
\hline 10 Semestre 1996 & $0,194^{* *}$ & $0,260^{\text {** }}$ \\
\hline 2 Semestre 1996 & $0,010^{*}$ & $0,005^{*}$ \\
\hline 10 Semestre 1997 & $0,128^{* *}$ & $0,553^{* *}$ \\
\hline $2^{\circ}$ Semestre 1997 & $0,797^{* *}$ & $0,247^{* *}$ \\
\hline 2o Semestre 1998 & 0,602 & 0,614 \\
\hline 2o Semestre 1999 & 0,374 & 0,466 \\
\hline
\end{tabular}

Obs.: $\left(^{*}\right)$ como p-value $<0,05$, as hipóteses nulas foram rejeitadas;

$\left(^{* *}\right)$ o Minitabß alertou que as amostras foram consideradas pequenas.

Uma vez que não foram detectadas diferenças significativas entre as carteiras, foi utilizado o conjunto das ações (elevando $\mathrm{n}$ e tornando os testes mais poderosos), para avaliar a diferença destas com o retorno do lbovespa nos períodos correspondentes.

Para esta comparação, procedeu-se ao teste de Wilcoxon - W que, analogamente ao teste KW acima, não depende de fortes suposições sobre a distribuição dos dados. Nesses casos, as diferenças foram consideradas pouco significativas, exceção feita ao primeiro semestre de 1997 ( $p=0,033)$, que apontou diferença de retorno entre o lbovespa e as 14 ações da amostra -4 da carteira de alto yield, 4 da carteira de baixo yield e 6 da carteira de zero yield (tabela 3.8).

Tabela 3.8 - Teste W para Comparação de Retornos com o lbovespa

\begin{tabular}{|l|c|}
\hline $\boldsymbol{P}$-value & Teste W \\
\hline 2 Semestre 1994 & 0,051 \\
\hline 1 Semestre 1995 & 0,950 \\
\hline 1 Semestre 1996 & 0,222 \\
\hline 1 Semestre 1997 & $0,033^{*}$ \\
\hline 2 Semestre 1997 & 0,508 \\
\hline 2 Semestre 1998 & 0,486 \\
\hline 2 Semestre 1999 & 0,616 \\
\hline
\end{tabular}

Obs.: (*) como p-value <0,05, a hipótese nula foi rejeitada.

Além desse teste, foi feito, em caráter experimental, um teste paramétrico sobre o retorno médio das ações visando compará-lo com o lbovespa. Em geral, as diferenças foram novamente consideradas pouco significativas, com exceção, agora, do $2^{\circ}$ semestre/1994 ( $p=0,019)$, quando foi apontada uma diferença entre o retorno das ações e do lbovespa (tabela 3.9).

Tabela 3.9 - Teste t para Comparação de Retornos com o lbovespa

\begin{tabular}{|l|c|}
\hline $\boldsymbol{P}$-value & Teste t \\
\hline 2 ○ Semestre 1994 & $0,019^{*}$ \\
\hline 1 Semestre 1995 & 0,540 \\
\hline 1 Semestre 1996 & 0,222 \\
\hline 1 Semestre 1997 & 0,950 \\
\hline $2^{\circ}$ Semestre 1997 & 0,350 \\
\hline $2 \circ$ Semestre 1998 & 0,570 \\
\hline $2 \circ$ Semestre 1999 & 0,610 \\
\hline
\end{tabular}

Obs.: $\left(^{*}\right)$ como p-value <0,05, a hipótese nula foi rejeitada. 
Cabe aqui um comentário sobre o ano de 1997. Naquele exercício, as ações preferenciais do Banespa contabilizaram retornos reais de $531 \%$ e $132 \%$ em cada semestre respectivo. Excluindo suas ações da análise, rejeitar-se-ia a hipótese nula para o primeiro semestre em ambos os testes $(t=0,0009 \mathrm{eW}=0,004)$. Para o segundo semestre, entretanto, as diferenças continuariam pouco significativas ( $t=0,93 \mathrm{eW}=0,776$ ).

As exceções à igualdade das taxas de retorno semestrais acumuladas ficaram por conta dos segundos semestres de 1995 e 1996 que, tanto pelo teste KW ( $p=0,02$ e 0,01$)$, quanto pela ANOVA ( $p=0,018$ e 0,005), rejeitaram-se as hipóteses nulas, significando que os desempenhos não foram os mesmos entre as carteiras naqueles períodos (tabela 3.7).

Para estes casos realizaram-se os testes par a par a fim de se identificarem as diferenças (tabela 3.10). No $2^{\circ}$ semestre/1995, foi observada diferença (teste de Mann-Whitney $-\mathrm{MW}=0,0065$ e $\mathrm{t}=0,0028$ ) entre os retornos da carteira de alto e de baixo yield. Já no oㅡ semestre/1996, além da diferença entre as carteiras de alto e de baixo yield ( $\mathrm{MW}=0,0332 \mathrm{e} \mathrm{t}=0,016)$, também foi detectada diferença entre as carteiras de alto e zero yield ( $\mathrm{MW}=0,0046 \mathrm{e} \mathrm{t}=0,0012$ ).

Como não foram detectadas diferenças entre os retornos das carteiras de baixo e de zero yield, procedeu-se à análise em conjunto para ambas as carteiras. No segundo semestre de 1995, o conjunto de ações de baixo e zero yield apresentou $\mathrm{W}=0,235 \mathrm{et}$ $=0,22$, enquanto, no segundo semestre de $1996, \mathrm{~W}=$ 0,295 e t $=0,24$, aceitando-se, para ambos, a hipótese nula de retornos iguais ao do lbovespa.

Tabela 3.10 -Teste Par a par para Diferenças de Retornos entre as Carteiras

\begin{tabular}{|c|c|c|c|c|c|c|c|}
\hline \multicolumn{2}{|c|}{ P-value } & \multicolumn{2}{c|}{ Alto Yield } & \multicolumn{2}{c|}{ Baixo Yield } & \multicolumn{2}{c|}{ Zero Yield } \\
\cline { 3 - 8 } \multicolumn{2}{|c|}{} & MW & t & MW & t & MW & t \\
\hline \multirow{2}{*}{ Alto Yield } & $2^{\circ}$ S/ 1995 & & & & & & \\
\cline { 2 - 8 } & $2^{\circ}$ S/ 1996 & & & & & & \\
\hline \multirow{2}{*}{ Baixo Yield } & $2^{\circ}$ S/ 1995 & $0,0065^{*}$ & $0,0028^{*}$ & & & & \\
\cline { 2 - 8 } & $2^{\circ}$ S/ 1996 & $0,0332^{*}$ & $0,0160^{*}$ & & & & \\
\hline Zero Yield & $2^{\circ}$ S/ 1995 & 0,7469 & 0,5000 & 0,0741 & 0,0770 & & \\
\hline & $2^{\circ}$ S/ 1996 & $0,0046^{*}$ & $0,0012^{*}$ & 0,7210 & 0,9800 & & \\
\hline
\end{tabular}

Obs.: $\left({ }^{*}\right.$ como $p$-value $<0,05$, a hipótese nula foi rejeitada.

Por sua vez, quando comparadas as carteiras em separado com o lbovespa, a fim de se observarem diferenças (tabela 4.11), exceto a carteira de alto yield, que apresentou diferenças no 20 semestre de $1995(\mathrm{~W}=0,014$ e $t=0,001)$ e no $2^{\circ}$ semestre de $1996(\mathrm{~W}=0,014$ e $\mathrm{t}=0,0034)$, as demais carteiras seguiram a maioria dos resultados apontados pelas tabelas 3.6 e 3.7, não apresentando diferenças estatisticamente significativas com o lbovespa.

Tabela 3.11 -Testes W e t para Comparação de Retornos com o lbovespa

\begin{tabular}{|l|c|c|c|}
\hline \multirow{2}{*}{ Alto Yield } & P-value & W & t \\
\cline { 2 - 4 } & $2 \circ \mathrm{S} / 1995$ & $0,014^{*}$ & $0,001^{*}$ \\
\hline \multirow{2}{*}{ Baixo Yield } & $2 \circ \mathrm{S} / 1996$ & $0,014^{*}$ & $0,0034^{*}$ \\
\cline { 2 - 4 } & $2 \circ \mathrm{S} / 1995$ & 1,00 & 0,76 \\
\hline \multirow{2}{*}{ Zero Yield } & $2 \circ \mathrm{S} / 1996$ & 0,675 & 0,57 \\
\cline { 2 - 4 } & $2 \circ \mathrm{S} / 1995$ & 0,142 & 0,096 \\
\cline { 2 - 4 } & $2 \circ \mathrm{S} / 1996$ & 0,353 & 0,23 \\
\hline
\end{tabular}

Obs.: $\left(^{\star}\right)$ como p-value $<0,05$, a hipótese nula foi rejeitada. 
Prejudicadas as análises, provavelmente em razão da grande variabilidade, procedeu-se à análise descritiva semestral das carteiras (tabelas 3.12, 3.13, 3.14 e 3.15$)$.

Tabela 3.12 - Retornos Semestrais Acumulados

\begin{tabular}{|c|c|c|c|c|}
\hline Percentual a.s. & Alto Yield & Baixo Yield & Zero Yield & Ibovespa \\
\hline 2 Semestre 1994 & 11,4 & $-2,5$ & 5,6 & $-9,9$ \\
\hline 10 Semestre 1995 & $-24,7$ & $-23,5$ & $-13,0$ & $-25,3$ \\
\hline 2 Semestre 1995 & $-17,5$ & 10,3 & $-15,7$ & 10,8 \\
\hline 10 Semestre 1996 & 21,6 & 26,7 & 27,0 & 27,9 \\
\hline 2 Semestre 1996 & $-1,8$ & 13,1 & 43,8 & 15,0 \\
\hline 1ㅇ Semestre 1997 & 11,6 & 48,5 & 94,5 & 69,0 \\
\hline 2 Semestre 1997 & $-11,0$ & $-23,9$ & $-12,6$ & $-20,1$ \\
\hline 10 Semestre 1998 & $-3,7$ & $-11,5$ & $-19,9$ & $-7,4$ \\
\hline 2 Semestre 1998 & $-21,3$ & $-30,6$ & $-32,6$ & $-29,3$ \\
\hline 10 Semestre 1999 & 81,7 & 119,5 & 102,3 & 64,3 \\
\hline 2o Semestre 1999 & 43,2 & 27,1 & 27,2 & 40,6 \\
\hline Acumulado Total & 61,7 & 128,5 & 233,6 & 123,5 \\
\hline
\end{tabular}

Na comparação semestral percebe-se que, em termos de retorno, a carteira zero yield obteve, na maioria das vezes, um desempenho melhor, não apenas em relação às outras carteiras, como também em relação ao Ibovespa (tabela 3.12 ) até o 1ㅇ semestre de 1997. Tanto em ciclos de alta como de baixa do mercado, a carteira zero yield obteve maio- res retornos durante aquele período.

Nota-se também que a carteira de baixo yield não só contabilizou um retorno acumulado muito próximo ao retorno do lbovespa (128,5\% vs. $123,5 \%$ ), mas também manteve, por mais tempo, altos níveis de correlação com o mercado (tabela 3.13).

Tabela 3.13 - Coeficientes de Correlação Semestrais

\begin{tabular}{|l|r|r|r|r|}
\multicolumn{1}{c|}{2 S/94 alto yield baixo yield Zero yield lbovespa } \\
\begin{tabular}{|l|r|r|r|r|}
\hline alto yield & 1 & & & \\
\hline baixo yield & 0,782142 & 1 & & \\
\hline zero yield & 0,991996 & 0,819452 & 1 & 1 \\
\hline lbovespa & 0,86879 & 0,964173 & 0,913644 & 1 \\
\hline
\end{tabular}
\end{tabular}

$1 S / 95$ alto yield baixo yield zero yield lbovespa

\begin{tabular}{|l|r|r|r|r|}
\hline alto yield & 1 & & & \\
\hline baixo yield & 0,777262 & 1 & & \\
\hline zero yield & 0,94268 & 0,745476 & 1 & \\
\hline Ibovespa & 0,811298 & 0,995364 & 0,753209 & 1 \\
\hline
\end{tabular}

$2 S / 95$ alto yield baixo yield zero yield lbovespa

\begin{tabular}{|l|r|r|r|r|}
\hline alto yield & 1 & & & \\
\hline baixo yield & 0,536114 & 1 & & \\
\hline zero yield & 0,793665 & 0,490835 & 1 & \\
\hline Ibovespa & 0,684699 & 0,953877 & 0,521718 & 1 \\
\hline
\end{tabular}

$1 S / 96$ alto yield baixo yield zero yield lbovespa

\begin{tabular}{|l|r|r|r|r|}
\hline alto yield & 1 & & & \\
\hline baixo yield & 0,654408 & 1 & & \\
\hline zero yield & 0,557246 & 0,772443 & 1 & \\
\hline Ibovespa & 0,814571 & 0,947436 & 0,807828 & 1 \\
\hline
\end{tabular}

$1 S / 97$ alto yield baixo yield zero yield lbovespa

\begin{tabular}{|l|r|r|r|r|}
\hline alto yield & 1 & & & \\
\hline baixo yield & 0,188119 & 1 & & \\
\hline zero yield & 0,151279 & 0,517669 & 1 & \\
\hline lbovespa & 0,249208 & 0,443245 & 0,419625 & 1 \\
\hline
\end{tabular}

2S/97 alto yield baixo yield zero yield lbovespa

\begin{tabular}{|l|r|r|r|r|}
\hline alto yield & 1 & & & \\
\hline baixo yield & 0,758292 & 1 & & \\
\hline zero yield & 0,810695 & 0,80287 & 1 & \\
\hline Ibovespa & 0,754572 & 0,985202 & 0,738992 & 1 \\
\hline
\end{tabular}

$1 S / 98$ alto yield baixo yield zero yield lbovespa

\begin{tabular}{|l|r|r|r|r|}
\hline alto yield & 1 & & & \\
\hline baixo yield & 0,973322 & 1 & & \\
\hline zero yield & 0,814207 & 0,823228 & 1 & \\
\hline Ibovespa & 0,978054 & 0,92777 & 0,760272 & 1 \\
\hline
\end{tabular}

2S/98 alto yield baixo yield zero yield lbovespa

\begin{tabular}{|l|r|r|r|r|}
\hline alto yield & 1 & & & \\
\hline baixo yield & 0,88079 & 1 & & \\
\hline zero yield & 0,759888 & 0,937053 & 1 & \\
\hline Ibovespa & 0,899701 & 0,88677 & 0,80653 & 1 \\
\hline
\end{tabular}




\begin{tabular}{|l|r|r|r|r|}
\hline \multicolumn{1}{|c|}{2 S/96 alto yield baixo yield zero yield lbovespa } \\
\hline alto yield & 1 & & & \\
\hline baixo yield & $-0,28743$ & 1 & & \\
\hline zero yield & 0,053972 & $-0,20569$ & 1 & \\
\hline Ibovespa & 0,625583 & 0,384549 & 0,296623 & 1 \\
\hline
\end{tabular}

Nos 11 semestres pesquisados, os coeficientes de correlação das carteiras com o lbovespa mostraramse positivos e, em boa parte, elevados. No intervalo mais longo de alta, entretanto, à medida que o índice de mercado alcançava os mais altos pontos, notam-se substanciais quedas nos coeficientes de correlação das carteiras com o mercado (2 semestre/1996 e 1ㅇ semestre/1997). Isto talvez se deva à suspeita de que, em ciclos de alta, a primeira onda de valorizações atinge, inicialmente, somente as blue chips, alastrando-se posteriormente às ações de segunda linha nobre, demais ações de segunda linha e, por fim, às ações de menor liquidez. Assim, quanto mais longo fosse o ciclo, mais distribuídas (e portanto, menos concentradas) estariam as altas de preços.

Salvo nos casos supramencionados, o único instante a apresentar uma correlação positiva fraca com o lbovespa foi no 1은 semestre/1999, com a carteira de zero yield, justamente o período em que esta carteira deteve o menor número de ações (3), pelo maior espaço de tempo consecutivo dentro de um mesmo semestre (4 meses).

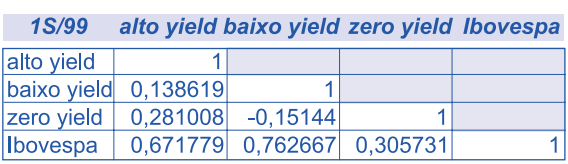

2S/99 alto yield baixo yield zero yield Ibovespa \begin{tabular}{|l|r|r|r|r|}
\hline alto yield & 1 & & & \\
\hline baixo yield & 0,815911 & 1 & & \\
\hline zero yield & 0,918337 & 0,876041 & 1 & \\
\hline Ibovespa & 0,819481 & 0,92253 & 0,73778 & 1 \\
\hline
\end{tabular}

Em nenhuma carteira se registraram coeficientes de correlação nulos. Os parcos coeficientes de correlação negativos foram observados no $2^{\circ}$ semestre / 1996 entre a carteira de baixo yield e as carteiras de alto $(\rho=-0,287)$ e zero $(\rho=-0,206)$ yields; e também no $1^{\circ}$ semestre/1999, novamente entre a carteira de baixo e a de zero yield $(\rho=-0,151)$; indicando que houve momentos de relação fraca e inversa entre as carteiras.

A tabela 3.14, por sua vez, reforça o fenômeno anteriormente verificado na tabela 3.12, de que toda a superioridade de retorno da carteira de zero yield esvaziou-se após o meio do ano de 1997.

Por outro lado, a carteira de alto yield, que teve as piores médias de retornos no ciclo de alta do $2^{\circ}$ semestre/1995 até o 1ํ semestre/1997, inverteu sua posição no ciclo de baixa que se seguiu até o $2^{\circ}$ semestre/1998, ou seja, apresentou as menores quedas médias, em um comportamento típico de carteiras com beta inferior a um; e ainda teve a maior alta média no último semestre do período de pesquisa (6,6 \% a.m.).

Tabela 3.14-Resultados por Semestre

\begin{tabular}{|c|c|c|c|c|c|c|c|c|}
\hline \multirow[t]{2}{*}{ Percentual a.m. } & \multicolumn{2}{|c|}{ Alto Yield } & \multicolumn{2}{|c|}{ Baixo Yield } & \multicolumn{2}{|c|}{ Zero Yield } & \multicolumn{2}{|c|}{ Ibovespa } \\
\hline & Retorno & Risco & Retorno & Risco & Retorno & Risco & Retorno & Risco \\
\hline 2o Semestre 1994 & 2,6 & 11,1 & 0,0 & 11,7 & 2,0 & 15,8 & $-1,4$ & 13,3 \\
\hline 10 Semestre 1995 & $-4,4$ & 7,3 & $-3,5$ & 14,9 & $-2,1$ & 7,3 & $-3,9$ & 15,1 \\
\hline 2 Semestre 1995 & $-3,0$ & 5,9 & 2,0 & 8,5 & $-2,5$ & 8,3 & 2,0 & 8,3 \\
\hline 10 Semestre 1996 & 3,8 & 12,1 & 4,2 & 5,9 & 4,4 & 9,5 & 4,4 & 7,6 \\
\hline 2o Semestre 1996 & $-0,2$ & 5,0 & 2,1 & 1,8 & 6,8 & 11,8 & 2,4 & 1,7 \\
\hline 1ㅇ Semestre 1997 & 1,9 & 5,0 & 6,9 & 3,6 & 12,1 & 10,2 & 9,2 & 3,9 \\
\hline 2 Semestre 1997 & $-1,4$ & 11,1 & $-3,7$ & 13,1 & $-1,8$ & 9,8 & $-2,7$ & 14,4 \\
\hline 1ㅇ Semestre 1998 & $-0,1$ & 11,7 & $-1,5$ & 10,8 & $-2,8$ & 14,9 & $-0,8$ & 10,2 \\
\hline 2o Semestre 1998 & $-2,6$ & 17,3 & $-4,7$ & 16,2 & $-4,1$ & 23,0 & $-3,1$ & 22,9 \\
\hline 10 Semestre 1999 & 11,0 & 12,6 & 14,7 & 14,8 & 13,2 & 14,3 & 8,9 & 8,7 \\
\hline 2 Semestre 1999 & 6,6 & 10,9 & 4,4 & 8,8 & 4,7 & 12,8 & 6,4 & 12,1 \\
\hline
\end{tabular}


Como, após o 1o semestre / 1997, os retornos foram negativos, a interpretação dos coeficientes semestrais de variação ficou prejudicada. Em relação à carteira de alto yield, pode-se destacar apenas que, nos primeiros semestres de 1996, 1997 e 1999, seus coeficientes foram piores que os demais, obtendo posição inversa no último semestre pesquisado (tabela 3.15).

Tabela 3.15 - Coeficientes de Variação

\begin{tabular}{|c|c|c|c|c|}
\hline Percentual a.s. & Alto Yield & Baixo Yield & Zero Yield & Ibovespa \\
\hline 2o Semestre 1994 & 422,7 & $\mathrm{R} / \mathrm{N}$ & 801,2 & $\mathrm{R} / \mathrm{N}$ \\
\hline 1ㅇ Semestre 1995 & $\mathrm{R} / \mathrm{N}$ & $\mathrm{R} / \mathrm{N}$ & $\mathrm{R} / \mathrm{N}$ & $\mathrm{R} / \mathrm{N}$ \\
\hline 2 Semestre 1995 & $\mathrm{R} / \mathrm{N}$ & 434,8 & $\mathrm{R} / \mathrm{N}$ & 413,1 \\
\hline 10 Semestre 1996 & 314,9 & 140,6 & 215,5 & 171,7 \\
\hline 2o Semestre 1996 & $\mathrm{R} / \mathrm{N}$ & 86,7 & 174,0 & 70,3 \\
\hline 1ㅇ Semestre 1997 & 258,0 & 52,6 & 84,1 & 42,2 \\
\hline 2 Semestre 1997 & $\mathrm{R} / \mathrm{N}$ & $\mathrm{R} / \mathrm{N}$ & $\mathrm{R} / \mathrm{N}$ & $\mathrm{R} / \mathrm{N}$ \\
\hline 10 Semestre 1998 & $\mathrm{R} / \mathrm{N}$ & $\mathrm{R} / \mathrm{N}$ & $\mathrm{R} / \mathrm{N}$ & $\mathrm{R} / \mathrm{N}$ \\
\hline 2o Semestre 1998 & $\mathrm{R} / \mathrm{N}$ & $\mathrm{R} / \mathrm{N}$ & $\mathrm{R} / \mathrm{N}$ & $\mathrm{R} / \mathrm{N}$ \\
\hline 10 Semestre 1999 & 114,6 & 100,7 & 108,0 & 97,7 \\
\hline 2 Semestre 1999 & 164,1 & 200,7 & 270,5 & 189,2 \\
\hline
\end{tabular}

$R / N$ - taxa de retorno negativa.

Na análise dos indicadores, novamente tanto o índice de Treynor quanto o de Sharpe mostraram que a carteira de alto yield teve o pior desempenho entre 1995 e 1997, sendo que o índice de Sharpe mostra uma reversão já no segundo semestre, enquanto o índice de Treynor só a capta no primeiro semestre de 1998, talvez por este último se utilizar de betas apurados anualmente (tabela 3.16).

Tabela 3.16 - Indicadores Semestrais de Desempenho

\begin{tabular}{|c|c|c|c|c|c|c|}
\hline \multirow[t]{2}{*}{ Indicadores } & \multicolumn{2}{|c|}{ Alto Yield } & \multicolumn{2}{|c|}{ Baixo Yield } & \multicolumn{2}{|c|}{ Zero Yield } \\
\hline & Treynor & Sharpe & Treynor & Sharpe & Treynor & Sharpe \\
\hline 20 Semestre 1994 & $-0,016$ & $-0,073$ & $-0,036$ & $-0,292$ & $-0,025$ & $-0,362$ \\
\hline 10 Semestre 1995 & -0.163 & $-1,104$ & $-0,077$ & $-0,484$ & $-0,099$ & $-0,502$ \\
\hline 2o Semestre 1995 & $-0,115$ & $-0,973$ & $-0,008$ & $-0,086$ & $-0,089$ & $-0,083$ \\
\hline 10 Semestre 1996 & 0,015 & 0,164 & 0,032 & 0,395 & 0,026 & 0,338 \\
\hline 20 Semestre 1996 & $-0,017$ & $-0,461$ & 0,000 & 0,002 & 0,048 & 0,174 \\
\hline 10 Semestre 1997 & 0,005 & 0,049 & 0,060 & 1,428 & 0,146 & 1,928 \\
\hline 2 Semestre 1997 & $-0,091$ & $-0,400$ & $-0,077$ & $-0,513$ & $-0,067$ & $-0,398$ \\
\hline 10 Semestre 1998 & $-0,037$ & $-0,237$ & $-0,061$ & $-0,391$ & $-0,064$ & $-0,348$ \\
\hline 2o Semestre 1998 & $-0,082$ & $-0,356$ & $-0,119$ & $-0,508$ & $-0,089$ & $-0,287$ \\
\hline 10 Semestre 1999 & 0,098 & 0,643 & 0,126 & 0,798 & 0,142 & 0,691 \\
\hline 2o Semestre 1999 & 0,067 & 0,508 & 0,035 & 0,374 & 0,050 & 0,438 \\
\hline
\end{tabular}

Contudo, resultados assim conflitantes são exceção. Na maioria das observações, ambos os indicadores parecem caminhar na mesma direção. Essas divergências, na opinião de ODA \& SECURATO (1996: 345), são notadas em carteiras de elevado risco próprio.
A recuperação da carteira de alto yield sofre apenas um revés na primeira metade do ano de 1999 (também captado pelo coeficiente de variação da tabela 3.13), quando seus indicadores de Treynor e Sharpe apontam um pior desempenho.

Enquanto os índices de Treynor e Sharpe são indi- 
cadores de desempenho relativo de carteiras com base no risco ajustado, o alfa de Jensen mede o desempenho absoluto de uma carteira. Graças à posição do intercepto $\alpha$ (que não precisa estar necessariamente na origem), permite-se avaliar a capacidade preditiva da estratégia (seleção de ações baratas ou subavaliadas).

Para o cálculo do alfa de Jensen foram comparadas as taxas de retorno mensais para todo o período de pesquisa (julho/1994 a dezembro/1999), com as taxas de retorno mensais do CDI Over. Assim, como na estatística descritiva, o $\alpha$ para as carteiras de alto $(-0,009)$ e baixo $(-0,002)$ yield indica desempenho inferior ao do mercado. A carteira de zero yield, por sua vez, foi capaz de contabilizar desempenho superior $(0,006)$, expressando um resultado maior que o mercado como um todo durante o período total.

\section{CONSIDERAÇÕES FINAIS}

A seleção de ações com base no critério de dividend yield e o posterior agrupamento dessas ações em carteiras serviram para auxiliar na comparação entre as taxas de retorno das ações selecionadas e 0 lbovespa.

Para tanto foram estabelecidas três hipóteses.

a) as médias de retornos apresentadas pela carteira de alto dividend yield e pelo Ibovespa são estatisticamente diferentes de zero;

b) as médias de retornos apresentadas pela carteira de baixo dividend yield e pelo lbovespa são estatisticamente diferentes de zero;

c) as médias de retornos apresentadas pela carteira de zero dividend yield e pelo lbovespa são estatisticamente diferentes de zero.

Se fosse considerado o desempenho das carteiras durante todo o período, ou seja, de ponta a ponta, a carteira composta de ações com alto dividend yield histórico não teria sido capaz de superar a taxa de retorno do índice de mercado, o lbovespa; ao passo que a carteira de zero yield apresentaria um retorno de cerca de 110 pontos percentuais superiores ao Ibovespa (tabela 3.2).

Tais diferenças foram, não obstante, consideradas não-significativas pelo teste estatístico aplicado (tabela 3.3).

Prosseguindo então a análise em bases semes- trais e de forma mais refinada, ou seja, através do desempenho de seus elementos (as ações), a tentativa de detectar novamente diferenças entre as médias dos retornos dentre as carteiras e entre estas e o Ibovespa.

Para se corroborarem as hipóteses desta pesquisa, contudo, era necessário - nesta etapa - saber inicialmente se as médias de retorno das ações das três carteiras (populações) são iguais.

Entre as carteiras foram detectadas diferenças entre as médias nos segundos semestres de $1995 \mathrm{e}$ 1996 (tabela 3.7), as quais foram identificadas pelos testes par a par como sendo as de alto e baixo yields (em ambos semestres) e as de alto e zero yields (no 2o semestre de 1996 apenas).

Porém nem os testes paramétricos nem os nãoparamétricos foram capazes de identificar diferenças estatisticamente significativas entre as carteiras e o Ibovespa, com apenas quatro exceções.

Para os casos em que as médias das carteiras foram consideradas iguais, ou seja, quando foram comparados os grupos de ações em conjunto (melhorando o tamanho da amostra $\mathrm{n}$ ), foram rejeitas as hipóteses nulas $\mathrm{H}_{0}$ :

a) no $2^{\circ}$ semestre/1994 $(\mathrm{n}=22)$, com $p$-value $=$ 0,019 para o t de Student e $p$-value $=0,051$ para Wilcoxon (muito próximo do limite do nível de significância $=0,05)$, quando então o Ibovespa registrou queda, as carteiras de zero e alto yield registraram alta e a carteira de baixo yield permaneceu estável;

b) no 1o semestre/1997 ( $\mathrm{n}=14)$, com $p$-value $=$ 0,033 para o teste de Wilcoxon, quando o Ibovespa superou as carteiras de alto e baixo yield, mas foi superado pela carteira de zero yield.

Já para a comparação entre carteiras e o lbovespa, rejeitaram-se as hipóteses de igualdade das médias dos retornos apenas para a carteira de alto yield com $\mathrm{n}=8$ nos períodos:

a) 2o semestre/1995, quando contabilizou queda frente a uma alta do lbovespa ( $p$-values de 0,014 e 0,001 para os testes de Wilcoxon e t, respectivamente);

b) $2^{\circ}$ semestre/1996, em situação idêntica ( $p$ values de 0,014 e 0,0034 para os testes de Wilcoxon e t, respectivamente).

Logo, salvo as exceções supramencionadas, os testes estatísticos não lograram detectar diferenças 
nas médias das taxas de retorno, nem entre as carteiras nem entre estas e o lbovespa.

Cabe ressaltar aqui que o reduzido tamanho das amostras e a elevada variabilidade das ações e, consequentemente, das carteiras podem ter exercido influência na aceitação das hipóteses nulas. Em outras palavras, isoladamente ou em conjunto, o número reduzido de elementos amostrais e a grande dispersão de suas médias possivelmente afetaram a nãorejeição das igualdades das médias, com a provável exceção da carteira de baixo yield, a ser discutida mais adiante.

Partindo para a análise das estatísticas descritivas, nota-se que, exceto pela elevada e positiva correlação entre a carteira de baixo dividend yield e o lbovespa para a maior parte do período, os resultados ponta a ponta apurados não foram capazes de apresentar padrões ou consistência nos desempenhos de nenhuma das carteiras.

Essa elevada correlação entre a carteira de baixo yield e o lbovespa, por sinal, foi provavelmente influenciada pela participação das ações da Telebrás (ordinárias e preferenciais) durante boa parte do seu período de avaliação.

Enquanto, por um lado, as ações ordinárias não estiveram presentes apenas em 17 dos 65 meses e as preferenciais somente em 6 meses; por outro lado, a alta concentração do lbovespa em alguns papéis durante o período de pesquisa, entre os quais Telebrás ON e PN (que ao final de 1999, por exemplo, representavam mais de $44 \%$ do referido índice de mercado), resultou em uma presença comum tanto no Ibovespa quanto na carteira de baixo yield por quase todo o período de pesquisa ${ }^{7}$.

Esta presença comum e quase constante ao índice e à carteira traz conseqüências à suposição de independência entre os grupos que, em princípio, havia estabelecido o lbovespa como elemento específico para fins comparativos.

Sabendo-se que as carteiras foram construídas a partir de ações que compõem o lbovespa (de modo a assegurar negociabilidade, liquidez e representatividade) e considerando-se o caráter não-experimental ou ex post facto deste estudo, não seria possível proceder à manipulação da referida variável ou à exclusão daque- las ações, sob pena de ignorar a realidade dos fatos tal qual se desenrolaram entre 1994 e 1999.

Outrossim, o lbovespa não estava concentrado à época em Telebrás por opção metodológica: apenas refletia a concentração do próprio mercado acionário, a qual decorre de conjunturas econômico-políticas (LEITE \& SANVICENTE, 1995: 62-67).

Quanto aos resultados "superiores" e "inferiores" das carteiras de zero e alto yield, respectivamente, estes não se mantiveram assim por todo o período de pesquisa. Ao contrário, parece ter havido um instante de "cisma" durante o ano de 1997, em que a carteira alto yield passou a registrar, de maneira geral, os maiores retornos e os menores riscos (tabelas 3.14 e 3.15).

Este ponto de mudança coincide com a eclosão da crise cambial na Tailândia, que rapidamente se alastrou para os outros países asiáticos e demais mercados emergentes ao redor do mundo (tabela 3.1).

Naquele ano, o mercado, refletido no comportamento do lbovespa, dava seqüência à tendência de alta que se iniciara no fim de 1995. Este movimento altista perdurou até julho de 1997, quando então o lbovespa contabilizou uma queda real de-17,4\%. A média diária de volume negociado girava em torno de $R \$ 1,2$ bilhão, sendo que os estrangeiros já respondiam por cerca de $1 / 3$ desse total.

Àquela altura, a Bovespa acumulava uma rentabilidade de quase $174 \%$ desde 1996 , dos quais $72,8 \%$ somente nos 7 primeiros meses de 1997; e também, pela primeira vez, o volume negociado com os american depositary receipts - ADRs da Telebrás na Bolsa de Valores de Nova lorque superava o volume negociado em São Paulo com as ações da mesma companhia.

Nos meses que se seguiram à crise, o Brasil deixou de apurar ingresso líquido de capitais, para registrar saídas líquidas de recursos. Somente nos meses de agosto, setembro e outubro daquele ano, o déficit foi de US\$2.018,7 milhões, forçando o Governo a tomar medidas para arrefecer os ânimos do mercado. $O$ Bacen vendeu $R \$ 1,1$ bilhão em títulos com correção cambial de 36 e 60 meses; o BNDESPar (Banco Nacional de Desenvolvimento Econômico e Social Participações) lançou $R$ \$ 330 milhões de opções de venda da Telebrás para fevereiro de 1998; enquanto a Bovespa

${ }^{7}$ Situações semelhantes foram encontradas nas carteiras de alto yield (Copene PNA) e zero yield (Cesp PN e Telesp ON), mas com pequenas participações no Ibovespa. 
instituía seu mecanismo de interrupção de negociação (circuit breaker) para o momento em que a queda do seu índice atingisse -10\% - Ofícios Circulares 355 e 359 (BOVESPA, 1997).
Separando o período de pesquisa em duas partes (até o primeiro semestre e após o segundo semestre de 1997), torna-se mais clara a observância desses dois momentos distintos (tabela 4.1).

Tabela 4.1 - Retorno e Risco para Dois Períodos

\begin{tabular}{|l|c|c|c|c|}
\hline & Alto Yield & Baixo Yield & Zero Yield & Ibovespa \\
\hline \multicolumn{5}{|c|}{ Retorno Acumulado \% a.p. } \\
\hline Jul /1994 - jun/1997 & $-7,9 \%$ & $75,2 \%$ & $175,1 \%$ & $85,1 \%$ \\
\hline Jul/1997 - dez/1999 & $75,5 \%$ & $30,5 \%$ & $21,3 \%$ & $20,7 \%$ \\
\hline \multicolumn{5}{|c|}{ Retorno Médio \% a.m. } \\
\hline Jul /1994 - jun/1997 & $0,1 \%$ & $2,0 \%$ & $3,5 \%$ & $2,2 \%$ \\
\hline Jul/1997 - dez/1999 & $2,7 \%$ & $1,8 \%$ & $1,8 \%$ & $1,7 \%$ \\
\hline \multicolumn{5}{|c|}{ Risco \% a.m. } \\
\hline Jul /1994 - jun/1997 & $8,1 \%$ & $8,8 \%$ & $11,1 \%$ & $9,7 \%$ \\
\hline Jul/1997 - dez/1999 & $13,1 \%$ & $14,1 \%$ & $15,9 \%$ & $14,4 \%$ \\
\hline \multicolumn{7}{|c|}{ Coeficiente de Variação \% } \\
\hline Jul /1994 - jun/1997 & $12.662,9 \%$ & $444,5 \%$ & $318,6 \%$ & $435,8 \%$ \\
\hline Jul/1997 - dez/1999 & $484,3 \%$ & $765,2 \%$ & $858,1 \%$ & $827,8 \%$ \\
\hline
\end{tabular}

Parte da explicação do melhor desempenho da carteira de zero dividend yield até meados de 1997 pode ser atribuída ao fato de que uma das ações que compunha a carteira auferiu uma taxa de retorno muito acima de qualquer outra, atuando como um outlier (observação aberrante ou discrepante) favorável à média dos retornos. Somente nos oito primeiros meses de 1997, antes de começar a cair, Banespa PN obteve um retorno real de quase $940 \%{ }^{8}$, um valor muito distante da média, o que contribuiu positivamente para o resultado da carteira de zero yield.

Por outro lado, de acordo com o citado na tabela 3.4, por falta de elementos, a carteira de zero yield foi incapaz de se beneficiar plenamente da diversificação durante boa parte daquele segundo momento do período de pesquisa. Entre novembro de 1997 e maio de 1999, a carteira não conseguiu ter mais do que 5 ações.

Para a carteira de alto yield, não foi possível identificar tão claramente um ou mais responsáveis (outliers) pelo pior desempenho no primeiro momento, nem pelo desempenho superior no segundo momento.
Dada a falta de consistência nos resultados (mesmo no segundo momento), não se pode afirmar que as ações de alto yield apresentem retornos médios significativamente superiores nem inferiores aos contabilizados por ações de outras categorias de dividend yield.

Como também não se observaram claramente padrões de desempenho superiores ou inferiores, nem que as taxas médias de retorno foram, em geral, significativamente diferentes em qualquer uma das carteiras ao retorno do Ibovespa, não se pode afirmar que as ações de alto ou baixo ou zero dividend yield são capazes de sobrepujar a taxa de retorno do índice de mercado.

Isto, por seu turno, implica que mesmo carteiras diversificadas (com aproximadamente dez ações), agrupadas conforme o yield, não têm motivos para apresentar retornos médios significativamente diferentes.

Assim, uma importante consideração final é que investidores que procuram "bater" o mercado devem ter em mente que não foram encontradas evidências empíricas que dêem suporte a quais-

\footnotetext{
${ }^{8}$ Vale lembrar que o desfecho da situação do banco (vide tabela 3.1) esteve incerto por vários meses, até que fosse declarada a intenção de privatizá-lo.
} 
quer estratégias de investimentos com base no yield das ações, isto é, não se deve esperar que investimentos em ações de alto ou baixo ou zero yields apresentem taxas de retorno capazes de superar o lbovespa.

Implicitamente, isto significa também que não se pode ratificar, portanto, o corolário de profissionais de mercado que acreditam que ações de alto dividend yield são capazes de gerar taxas de retorno significativamente superiores, quer em relação ao índice de mercado, quer em relação às ações de baixo ou zero yields.

Retomando o escopo principal que guiou esta pesquisa, isto é, a investigação da relação entre dividend yields e taxas de retorno das ações, o presente estudo não foi capaz de revelar que diferenças no dividend yield resultam em diferenças nas taxas de retorno.

Todavia, os resultados corroboram a conclusão de BLACK \& SCHOLES (1974), apurada em época, local, e com material e metodologia distintas, segundo a qual não foram encontradas evidências de que os retornos das ações de alto dividend yield são diferentes daqueles apurados por ações de baixo dividend yield, nem o efeito que os dividend yields exercem nas taxas de retornos das ações.

Dentro das limitações e delimitações deste trabalho, isso significa que a taxa de retorno esperada antes dos impostos de uma ação de alto dividend yield histórico, por si só, não tem motivos para ser significativamente diferente de uma ação que apresente baixo ou nulo dividend yield.
Nem mesmo com relação ao subproduto das taxas de retorno e sinônimo de risco segundo a MTP, o desvio padrão, podem ser extraídas assertivas de comportamento.

Além das considerações finais, que podem ter ampliado o conhecimento sobre o mercado de capitais brasileiro, bem como ter desmistificado (ainda que parcialmente) mais uma notória "crença" de mercado, o presente trabalho espera ter aberto espaço para futuras investigações científicas relacionadas ao tema, algumas das quais são mencionadas a seguir.

Inicialmente, na pesquisa realizada foram utilizados os dividend yields históricos como indicadores de retorno das ações. Obviamente, o comportamento passado dos dividendos por ação nem sempre se repetirá no futuro, o que inspira a projeção de tais valores. Uma vez que não foi realizado tal procedimento, fica estabelecido um quadro de como se comportam as taxas de retorno das ações em relação a dividend yields futuros.

Outro espaço igualmente de interesse acadêmico diz respeito à associação entre as taxas de retorno das ações, as categorias de dividend yield e os níveis de distribuição de resultados (payout ratios). Além da estratégia em epígrafe, seria avaliado conjuntamente se aumentos no percentual de lucro distribuído produzem elevações tão grandes nos preços das ações, capazes de resultar em quedas no yield. Investigar-se-ia, então, se ações de alto dividend yield possuem níveis de distribuição de resultados, inferiores às ações de baixo yield e assim por diante.

\section{REFERÊNCIAS BIBLIOGRÁFICAS}

BACHELIER, Louis. Théorie de la Speculation. Paris: Tese (Doutorado) - Faculdade de Ciências da Academia de Paris, Gauthier-Villars, 1900. in COOTNER (loc. cit.).

BERNSTEIN, Peter L. Capital Ideas. New York: The Free Press, 1992.

BLACK, Fischer; SCHOLES, Myron. The Effects of Dividend Yield and Dividend Policy on Common Stock Prices and Returns. Journal of Financial Economics. Amsterdam: North-Holland, v.1, n.1, p. 1-22, 1974.

BLUME, Marshall E. Stock Returns and Dividend Yields: some more evidence. The Review of Economics and Statistics. Amsterdam: North-Holland, v. 62, n.4, p. 567577, nov. 1980 .
BOLSA DE VALORES DE SÃO PAULO - BOVESPA. Revista Bovespa. São Paulo, ano IV, n.46, p.8-15, jul.1997.

BRIGHAM, Eugene F.; GORDON, Myron J. Leverage, Dividend Policy, and the Cost of Capital. The Journal of Finance. New York: American Finance Association, v. 23, n.1, p.85-103, mar.1968.

BUENO, Artur Franco. Análise Empírica do Dividend Yield das Ações Brasileiras. São Paulo: Dissertação (Mestrado) - Faculdade de Economia, Administração e Contabilidade da Universidade de São Paulo FEA/USP, 2000.

CHRISTIE, William G. Dividend Yield and Expected Returns - the zero-dividend puzzle Journal of Financial Economics. Amsterdam: North-Holland, v.6, n.1/2, p. 95-126, nov.-dez.1990. 
COOTNER, Paul H. The Random Character of Stock Market Prices. Cambridge: MIT Press, 1964.

ELTON, Edwin J.; GRUBER, Martin J.; RENTZLER, Joel. A Simple Examination of the Empirical Relationship Between Dividend Yields and Deviations from the CAPM. Journal of Banking and Finance. Amsterdam: North-Holland, v.7, n.1, p. 135-146, mar.1983.

FRIEND, Irwin; PUCKETT, Marshall. Dividends and Stock Prices. The American Economic Review. Menasha: The American Economic Association, v.54, n.5, p. 656682, set.1964.

GITMAN, Lawrence J. Princípios de Administração Financeira. 7.ed. São Paulo: Harbra, 1997.

GRAHAM, Benjamin; DODD, David L.; COTTLE, Sidney. Security Analysis: principles and technique. 4ed. New York: McGraw-Hill, 1962.

HESS, Patrick J. The Ex-dividend Day Behavior of Stock Returns: further evidence on tax effects. The Journal of Finance. New York: The American Finance Association, v.37, n.2, p.445-456, mai.1982.

KEIM, Donald B. Dividend Yields and Stock Returns implications of abnormal january results. Journal of Financial Economics. Amsterdam: North-Holland, v.14, n.3, p. 473-489, set.1985.

KEOWN, Arthur J.; SCOTT JR., David F.; MARTIN, John D.; PETTY, J. William. Basic Financial Management. 7ed. Upper Saddle River: Prentice-Hall, 1996.

KRAMER, Charles F. Explaining the Dividend Yield in the United States. Finance \& Development. Washington: Fundo Monetário Internacional - FMI, p. 24-25, dez.1997.

LEITE, Hélio de Paula; SANVICENTE, Antonio Zoratto. Índice Bovespa: um padrão para os investimentos brasileiros. São Paulo: Atlas, 1995.

LITZENBERGER, Robert H.; RAMASWAMY, Krishna. The Effect of Personal Taxes and Dividends on Capital Asset Prices. Journal of Financial Economics. Amsterdam: North-Holland, p. 163-195, n.2, v.7, jun.1979.

- Dividends, Short Selling Restrictions, Taxinduced Investor Clienteles and Market Equilibrium. Journal of Finance. New York: The American Finance Association, v.35, n.2, p.469482, mai. 1980.

. The Effects of Dividends on Common Stock Prices: tax effects or information effects? Journal of Finance. New York: The American Finance Association, v.37, n.2, p.429-443, mai.1982.

LONG JR., John B. The Market Valuation of Cash Dividends: a case to consider. Journal of Financial Economics.
Amsterdam: North-Holland, v.6, n.2/3, p. 235-264, jun.set.1978.

MEGLIORINI, Evandir. Lucros Distribuídos - fator de atratividade para investimento em ações. Osasco: Fundação Instituto Tecnológico de Osasco - FITO, 1998.

MILLER, Merton H.; MODIGLIANI, Franco. Dividend Policy, Growth, and the Valuation of Shares. The Journal of Business, v.34, p.411-433, out.1961 in VAN HORNE (loc. cit.).

MILLER, Merton H.; SCHOLES, Myron S. Dividends and Taxes: some empirical evidence. Journal of Political Economy. Chicago: University of, v.90, n.6, p. 11181141, dez.1982.

MORGAN, I.G. Dividends and Capital Asset Prices. The Journal of Finance. New York: The American Finance Association, v.37, n.4, p.1071-1086, set.1982.

ODA, André Luiz; SECURATO, José Roberto. Avaliação da Performance de Fundos - o índice de Elton e Gruber. Seminários em Administração - I SEMEAD. São Paulo: Programa de Pós-graduação em Administração do Departamento de Administração da Faculdade de Economia, Administração e Contabilidade da Universidade de São Paulo - FEA/USP, p. 340356, $15-16$ out.1996.

OSBORNE, M. F. M. Brownian Motion in the Stock Market. Operations Research, v.7, p. 145-173, mar.-abr.1959 in COOTNER (loc.cit.).

POTERBA, James M. The Market Valuation of Cash Dividends: the Citizens Utilities case reconsidered. Journal of Financial Economics. Amsterdam: NorthHolland, v.15, n.3, p.395-405, mar.1986.

ROBERTS, Harry V. Stock Market "Patterns" and Financial Analysis: methodological suggestions. The Journal of Finance. New York: The American Finance Association, v.14, n.1, p. 1-10, mar.1959.

ROSS, Stephen A.;WESTERNFIELD, Randolph W.; JAFFE, Jeffrey F. Administração Financeira. São Paulo: Atlas, 1995.

SPERANZINI, Milton Medeiros. Efeito da Política de Dividendos Sobre o Valor das Ações no Mercado Brasileiro de Capitais. São Paulo: Dissertação (Mestrado) - Faculdade de Economia, Administração e Contabilidade da Universidade de São Paulo - FEA/USP, 1994.

VAN HORNE, James C. Foundations for Financial Management. 2.ed. Homewood: Richard D. Irwin, 1967.

WESTON, J. F.; BRIGHAM, Eugene F. Fundamentos da Administração Financeira. 10.ed. São Paulo: Makron Books, 2000. 